\title{
New Ultrasonic Techniques for Colorectal Cancer Imaging
}

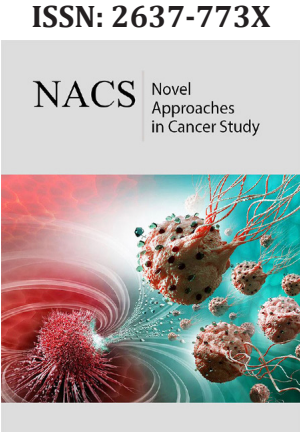

*Corresponding author: Rossana Soletti, Departamento Interdisciplinar, Federal University of Rio Grande do Sul, Tramandaí, Brazil

Submission: 盢 June 18, 2019

Published: 悳June 24, 2019

Volume 3 - Issue1

How to cite this article: Rossana Solleti, João Carlos M. New Ultrasonic Techniques for Colorectal Cancer Imaging. Nov Appro in Can Study. 3(1). NACS.000552.2019. DOI: 10.31031/NACS.2019.03.000552

Copyright@ Rossana Soletti, This article is distributed under the terms of the Creative Commons Attribution 4.0 International License, which permits unrestricted use and redistribution provided that the original author and source are credited.

\author{
Rossana Solleti ${ }^{1 *}$ and João Carlos Machado Ma,3 $^{2,3}$ \\ ${ }^{1}$ Interdisciplinar Department, Federal University of Rio Grande do Sul, Tramandaí, Brazil \\ ${ }^{2}$ Biomedical Engineering Program, COPPE, Federal University of Rio de Janeiro, Rio de Janeiro, \\ RJ, Brazil
}

${ }^{3}$ Post-Graduation Program in Surgical Sciences, Department of Surgery, School of Medicine, Federal University of Rio de Janeiro, Rio de Janeiro, RJ, Brazil

\begin{abstract}
Colorectal cancer (CRC) is the third most common cancer and the second cancer-related cause of mortality in the world, with increasing incidence in some developing countries and in the young population. Colonoscopy is the gold standard tool for CRC screening because it can detect and remove precancerous lesions in the same procedure, and its widespread use in people older than 50 years of age may be the cause of declining CRC incidence in this group. However, some precancerous lesions may be missed by colonoscopy and progress to CRC before the next screening, a fact that demonstrates the need to search for new affordable and sensitive imaging techniques. In this minireview we highlight the main ultrasound methods that have been studied experimentally and clinically with the potential to improve CRC detection, diagnosis and follow-up.
\end{abstract}

Keywords: Colorectal neoplasms; Diagnostic imaging; Ultrasonography; Ultrasound bio microscopy

\section{Introduction}

Colorectal cancer (CRC) is the third most common cancer diagnosed in the world and the second cancer-related cause of mortality in both men and women [1]. Despite of being about three times more incident in transitioned countries, the mortality rates for CRC are higher in transitioning countries, and these two discrepancies reflect that improvements in survival are due to the adoption of best practices in cancer treatment and management [2]. CRC presents a variation of incidence trends in different geographic regions: increasing incidence in some countries (such as China, Russia, Canada, The United Kingdom and Brazil) and decreasing incidence in others (including The United States, France and Japan) [3]. However, the overall declines in CRC incidence in countries such as The United States are masking an increasing incidence in young adults: from the mid- 1980 through 2013, rates of CRC incidence increased by $2.4 \%$ per year in adults aged $20-29$ years and by $1.0 \%$ per year in adults aged $30-39$ years [4]. It is estimated that by 2030 the incidence rates for colon and rectal cancer in the US population will increase by $90 \%$ and $124 \%$, respectively, for patients 20 to 34 years of age [5]. The declining CRC incidence in groups aged older than 50 years may be a reflect from the widespread screening in this population, which rose from 38\% in 2000 to 59\% in 2013 [6]. Fecal occult blood tests, flexible sigmoidoscopy and colonoscopy were the most common screening test modalities among older adults in The United States until 2005 [7]. By 2005, colonoscopy had become the most common imaging screening test modality for CRC in older adults [7], with its use among US adults aged 50 years and older reaching 60\% in 2015 [8].

\section{CRC Diagnostic}

The U.S. Multi-Society Task Force of Colorectal Cancer recommended CRC screening to average-risk persons (persons without a family history of colorectal neoplasia) beginning at age 50 years with colonoscopy being offered first [9]. Currently, the American Cancer Society recommends regular CRC screening for adults aged 45 and older with either a high-sensitivity stool-based test or an imaging exam [10]. Screening aims to reduce CRC incidence and mortality by removal of adenomatous polyps and by detection and treatment of early-stage cancers [11-12]. Usually, CRC arises from adenomas in an "adenoma-carcinoma sequence", a series of histopathological events associated with molecular alterations that can take more than 10 years to complete. In the Western population, the incidence of polyps among people aged 50 or older is $22-54 \%$ [13-14]. Moreover, about 30\% of diagnosed CRC had evolved from serrated lesions, which can be classified as hyperplastic polyps (not considered precancerous), 
sessile serrated polyps and traditional serrated adenomas [9]. In these cases, colonoscopy has the advantage to detect and remove precancerous lesions in the same procedure, reaching an estimated $53 \%$ reduction in CRC-induced mortality rates [11].

In terms of imaging modality tools for detection of all precancerous colorectal lesions, colonoscopy is still the gold standard one, being the most commonly performed procedure for CRC screening and surveillance in the United States [11]. Colonoscopy is advantageous in providing a clear visualization of the intestinal mucosal surface and in detecting and preventing CRC through polypectomy. However, since colonoscopy performs a superficial inspection of the mucosa, information from the other colonic layers are missed. Moreover, some patients diagnosed with CRC after a clear recent colonoscopy discloses the fact that a percentage of precancerous lesions could be undetected during the procedure. It is estimated that up to $6 \%$ of all CRC cases are caused by undetected polyps that progressed during the period between two colonoscopic procedures [15]. In a multicohort analysis from North American studies, 9167 participants who had adenomas removed during a first colonoscopy were followed until the subsequent colonoscopic procedure. From the 58 interval CRC detected cases, $52 \%$ were classified as resultant from probably missed lesions [16], suggesting that nearly half of interval colorectal cancers could be attributed to undetected lesions. In a study evaluating 314,872 colonoscopies performed by 136 gastroenterologists between 1998 and 2010, each 1\% increase in the adenoma detection rate was associated with a $5 \%$ decrease in the risk of a fatal interval CRC [14], therefore indicating that missed adenomas during colonoscopy increase the CRC incidence and mortality. A recent meta- analysis estimated the general adenoma miss rate of tandem colonoscopies as $21 \%$, when no auxiliary technique was employed in both procedures, and as $29 \%$ when the second colonoscopy was performed with an auxiliary technique [17]. These two findings claim that current colonoscopy auxiliary methods can improve the adenoma detection rate and, therefore, there is a need to search for new affordable and sensitive imaging methods to improve CRC diagnosis and follow-up.

\section{Role of Ultrasound Imaging in CRC Diagnosis}

Ultrasound (US) is an imaging technique that carries the advantages of being affordable, widely available, able to provide real time images and of not using ionizing radiation. Conventional transabdominal ultrasound can be used to detect colonic diseases and CRC, although it is not the first choice screening method, because it has limited access to the entire colon, caused by tissues and organs interposed between the colon and the probe that block the transmission of the US beam. To overcome this limitation, an endoluminal US transducer can be used in the detection and staging of colorectal lesions. When used to assess muscularis propria invasion for rectal cancer staging, the endoluminal ultrasonography has a specificity higher than that of magnetic resonance $(86 \%$ and $69 \%$, respectively) [18]. Endoluminal US can also be combined with colonoscopy, forming the endoscopic US (EUS) imaging technique. For rectal tumors, EUS and magnetic resonance imaging have been considered the standard staging modalities for several years [1920] and for early T1 cancers with an option for local excision, EUS is the first choice because of its superior near-field resolution [21].

Using a standard US transducer with a frequency bandwidth of $3.5-17 \mathrm{MHz}$, it is possible to distinguish all concentric layers of the rectal wall: the first echogenic layer is the Epithelium/mucosae, followed by a hypoechoic layer of muscularis mucosae, an echogenic ring of submucosa, an hypoechoic layer of muscularis propria and finally the hyperechogenic ring of adventicia/serosa [22]. In order to improve ultrasonic imaging resolution and accurately detect CRC depth, higher US frequencies must be used and that was the case of using $20 \mathrm{MHz}$ frequency endoluminal US to determine, accurately, if gastrointestinal tumors were restricted to the mucosa and submucosa layers [23-25]. Ultrasonic techniques using even higher frequencies (40-50MHz), named ultrasound biomicroscopy (UBM), are able to perform imaging of living tissues with near microscopic resolution. UBM using miniaturized endoluminal transducers, named endoluminal UBM (eUBM) and performed simultaneously with colonoscopy has been evaluated in the detection and followup of colorectal lesions in murine models of CRC [26-27]. eUBM presented a sensitivity superior to colonoscopy in the detection of colorectal lesions (0.93 and 0.83 , respectively) [26] and was able to correctly detect all colonic tumors and lymphoid infiltrates during follow-up [27], suggesting that high frequency endoluminal US could be used combined to colonoscopy, improving polyp detection rates.

US can be associated to ultrasound contrast agents (UCA), usually microbubbles $(1-7 \mu \mathrm{m})$ injected in a peripheral vein, which appear in the target tissue, increasing echogenicity and improving imaging delineation. The combined use of EUS with UCA introduces an image modality named CEUS (contrast-enhanced ultrasound) with improved imaging contrast that allows the visualization of perfusion, enabling real-time assessment and quantification of the colonic microvasculature. UCA flow inside the microvasculature can be measured as time-intensity curves (TIC), based on the time for wash-in and wash-out, allowing to determine the time to peak enhancement and the amount of enhancement [28]. In a clinical evaluation with 51 patients, transabdominal CEUS was able to differentiate between inflammatory bowel diseases and CRC, since colon cancer tissue showed later enhancement and slower wash-out with less speed to reach peak intensity [29]. The ability of a contrast-enhanced endoscopic ultrasound (CE- EUS) to assess vascular perfusion patterns in CRC tissue was also clinically evaluated in 42 patients. In this case, parameters generated from the TIC analysis could predict tumoral $\mathrm{N}$ staging and microvascular density, confirmed by CD31 immunostaining, suggesting that CE-EUS represents a feasible imaging technique for real-time angiogenesis measurement that may help in the choice of firstline therapy and the establishment of prognosis [30]. An emerging area of ultrasound imaging is the conjugation of UCAs to antibodies directed to target specific proteins involved in tumorigenesis and inflammation, enabling real-time protein expression measurements. To evaluate CRC vascularization, several studies have associated transabdominal US with Vascular Endothelial Growth Factor 
receptor 2 (VEGFR2)-targeted UCAs in murine models, achieving accurate assessments of tumor angiogenesis [31-34].

Photoacoustic (PA) imaging combines laser and ultrasound: transmitted nanosecond pulses of laser light (near infrared or visible) into tissue to yields rapid thermoelastic expansion that emits broadband ultrasound pulses. These pulses are received by an ultrasound transducer to construct a PA image, with contrast determined by optical absorption of tissue components such as hemoglobin, melanin, water or lipids. Because the hemoglobin in blood is a strong optical absorber, PA is suitable for imaging the vasculature and oxygenation, so the magnitude of ultrasonic emission reveals physiological patterns of biological tissues [35]. Recently, a photoacoustic microscopy with acoustic resolution (ARPAM) was able to detect the typical disorganized tissue structure and distorted vascular distribution of human CRC fresh tissue, in an ex vivo analysis, suggesting that PA imaging may assist in CRC diagnosis and therapy monitoring in the future [36].

\section{Conclusion}

Current CRC screening techniques based mainly on colonoscopy has halved the mortality of CRC, which nevertheless figures as the second most deadly cancer. Ultrasonic waves have been used in the development of new auxiliary imaging techniques aiming to improve the detection and diagnosis of CRC. In this context, photoacoustic and endoluminal high frequency ultrasound that can be associated to colonoscopy and ultrasound contrast agents, have become promising new tools to accurately detect, diagnose and stage CRC, as well as to monitor tumor vascularization.

\section{Acknowledgement}

The authors are thankful for the financial support received from the National Council for Scientific and Technological Development (CNPq), the Brazilian Federal Agency for Support and Evaluation of Higher Education (CAPES) and the Carlos Chagas Filho Foundation for Research Support of the State of Rio de Janeiro (FAPERJ).

\section{Conflict of Interest Statement}

None declared.

\section{References}

1. Ferlay J, Colombet M, Soerjomataram I, Mathers C, Parkin DM, et al. (2019) Estimating the global cancer incidence and mortality in 2018 GLOBOCAN sources and methods. Int J Cancer 144(8): 1941-1953.

2. Bray F, Ferlay J, Soerjomataram I, Siegel RL, Torre LA, et al. (2018) Global cancer statistics 2018: GLOBOCAN estimates of incidence and mortality worldwide for 36 cancers in 185 countries. CA Cancer J Clin 68(6): 394424.

3. Arnold M, Sierra MS, Laversanne M, Soerjomataram I, Jemal A, et al. (2017) Global patterns and trends in colorectal cancer incidence and mortality. Gut 66(4): 683-691.

4. Siegel RL, Fedewa SA, Anderson WF, Miller KD, Ma J, et al. (2017) Colorectal cancer incidence patterns in the United States, 1974-2013. J Natl Cancer Inst 109(8).

5. Bailey CE, Hu CY, You YN, Bednarski BK, Rodriguez-Bigas MA, et al. (2015) Increasing disparities in the age-related incidences of colon and rectal cancers in the United States, 1975-2010. JAMA Surg 150(1): 17 22.
6. Centers for disease control and prevention, national center for health statistics. National Health Interview Surveys 2000 and 2013. Public use data files 2001, 2014.

7. Chen X, White MC, Peipins LA, Seeff LC (2008) Increase in screening for colorectal cancer in older Americans: results from a national survey. J Am Geriatr Soc 56(8): 1511-1516.

8. Siegel RL, Miller KD, Jemal A (2019) Cancer statistics. CA Cancer J Clin 69(1): 7-34.

9. Rex DK, Ahnen DJ, Baron JA, Batts KP, Burke CA, et al. (2012) Serrated lesions of the colorectum: review and recommendations from an expert panel. Am J Gastroenterol 107(9): 1315-1329.

10. Wolf AMD, Fontham ETH, Church TR, Flowers CR, Guerra CE, et al. (2018) Colorectal cancer screening for average-risk adults: 2018 guideline update from the American Cancer Society. CA Cancer J Clin 68(4): 250281.

11. Zauber AG, Winawer SJ, O’Brien MJ, Lansdorp-Vogelaar I, van Ballegooijen $\mathrm{M}$, et al. (2012) Colonoscopic polypectomy and long-term prevention of colorectal- cancer deaths. N Engl J Med 366(8): 687-696.

12. McPhail S, Johnson S, Greenberg D, Peake M, Rous B (2015) Stage at diagnosis and early mortality from cancer in England. Br J Cancer 112(Suppl 1): S108-S115.

13. Imperiale TF, Wagner DR, Lin CY, Larkin GN, Rogge JD, et al. (2000) Risk of advanced proximal neoplasms in asymptomatic adults according to the distal colorectal findings. N Engl J Med 343(3): 169-174.

14. Corley DA, Jensen CD, Marks AR, Zhao WK, Lee JK, et al. (2014) Adenoma detection rate and risk of colorectal cancer and death. N Engl J Med 370(26): 1298-1306.

15. Bressler B, Paszat LF, Chen Z, Rothwell DM, Vinden C, et al. (2007) Rates of new or missed colorectal cancers after colonoscopy and their risk factors: a population-based analysis. Gastroenterology 132(1): 96-102.

16. Robertson DJ, Lieberman DA, Winawer SJ, Ahnen DJ, Baron JA, et al. (2014) Colorectal cancers soon after colonoscopy: a pooled multicohort analysis. Gut 63(6): 949-956.

17. Zhao S, Wang S, Pan P, Xia T, Chang X, et al. (2019) Magnitude, risk factors and factors associated with adenoma miss rate of tandem colonoscopy: A systematic review and meta-analysis. Gastroenterology 156(6): 16611674.

18. Bipat S, Glas AS, Slors FJ, Zwinderman AH, Bossuyt PM, et al. (2004) Rectal cancer: local staging and assessment of lymph node involvement with endoluminal US, CT, and MR imaging--a meta-analysis. Radiology 232(3): 773-783.

19. Wald C, Scheirey CD, Tran TM, Erbay N (2006) An update on imaging of colorectal cancer. Surg Clin North Am 86(4): 819-847.

20. Hasak S, Kushnir V (2019) Rectal endoscopic ultrasound in clinical practice. Curr Gastroenterol Rep 21(4): 18.

21. Lutz MP, Zalcberg JR, Glynne-Jones R, Ruers T, Ducreux M, et al. (2016) Second St. Gallen European Organisation for research and treatment of cancer gastrointestinal cancer conference: consensus recommendations on controversial issues in the primary treatment of rectal cancer. Eur J Cancer 63: 11-24.

22. Bor R, Fábián A, Szepes Z (2016) Role of ultrasound in colorectal diseases. World J Gastroenterol 22(43): 9477-9487.

23. Waxman I, Saitoh Y, Raju GS, Watari J, Yokota R, et al. (2002) Highfrequency probe EUS-assisted endoscopic mucosal resection: a therapeutic strategy for submucosal tumors of the GI tract. Gastrointest Endosc 55(1): 44-49.

24. Hunerbein M, Handke T, Ulmer C, Schlag PM (2004) Impact of miniprobe ultrasonography on planning of minimally invasive surgery for gastric and colonic tumors. Surg Endosc 18(4): 601-605. 
25. Hurlstone DP, Cross SS, Sanders DS (2005) 20MHz high-frequency endoscopic ultrasound-assisted endoscopic mucosal resection for colorectal submucosal lesions: a prospective analysis. J Clin Gastroenterol 39(7): 596-599.

26. Alves KZ, Soletti RC, de Britto MA, de Matos DG, Soldan M, et al. (2013) In vivo endoluminal ultrasound biomicroscopic imaging in a mouse model of colorectal cancer. Acad Radiol 20(1): 90-98.

27. Soletti RC, Alves KZ, de Britto MA, de Matos DG, Soldan M, et al. (2013) Simultaneous follow-up of mouse colon lesions by colonoscopy and endoluminal ultrasound biomicroscopy. World J Gastroenterol 19(44): 8056-8064.

28. Roccarina D, Garcovich M, Ainora ME, Caracciolo G, Ponziani F, et al. (2013) Diagnosis of bowel diseases: the role of imaging and ultrasonography. World J Gastroenterol 19(14): 2144-2153.

29. Zhang F, Miao LY, Ge HY, Tan S, Li ZQ et al. (2018) Usefulness of contrastenhanced ultrasound in differentiating inflammatory bowel disease from colon cancer. Ultrasound Med Biol 44(1): 124-133.

30. Cartana ET, Gheonea DI, Cherciu IF, Streaţa I, Uscatu CD, et al. (2018) Assessing tumor angiogenesis in colorectal cancer by quantitative contrast-enhanced endoscopic ultrasound and molecular and immunohistochemical analysis. Endosc Ultrasound 7(3): 175-183.

31. Wang H, Kaneko OF, Tian L, Hristov D, Willmann JK (2015) Threedimensional ultrasound molecular imaging of angiogenesis in colon cancer using a clinical matrix array ultrasound transducer. Invest Radiol 50(5): 322-329.
32. Zhou J, Wang H, Zhang H, Lutz AM, Tian L, et al. (2016) VEGFR2targeted three-dimensional ultrasound imaging can predict responses to antiangiogenic therapy in preclinical models of colon cancer. Cancer Res 76(14): 4081-4089.

33. Eschbach RS, Clevert DA, Hirner-Eppeneder H, Ingrisch M, Moser $M$, et al. (2017) Contrast-enhanced ultrasound with vegfr2targeted microbubbles for monitoring regorafenib therapy effects in experimental colorectal adenocarcinomas in rats with dce-mri and immunohistochemical validation. PLoS One 12(1): e0169323.

34. Turco S, El Kaffas A, Zhou J, Lutz AM, et al. (2018) Pharmacokinetic modeling of targeted ultrasound contrast agents for quantitative assessment of anti- angiogenic therapy: a longitudinal case-control study in colon cancer. Mol Imaging Biol.

35. Beard P (2011) Biomedical photoacoustic imaging. Interface Focus 1(4): 602-631.

36. Leng X, Chapman W Jr, Rao B, Nandy S, Chen R, et al. (2018) Feasibility of co- registered ultrasound and acoustic-resolution photoacoustic imaging of human colorectal cancer. Biomed Opt Express 9(11): 51595172. 